\title{
Co-occurrence network of TV advertisements revealing Japanese lifestyle
}

\author{
Mariko I. Ito* (10) and Takaaki Ohnishi
}

\section{${ }^{*}$ Correspondence:}

marikoito.rnu1@gmail.com Graduate School of Artificial Intelligence and Science, Rikkyo University, Tokyo, Japan

\begin{abstract}
The relationship between culture and the appeals in TV advertisements has been extensively studied. We attempted to reveal the image structure produced by TV commercials in Japan, which may show the cultural features of the country, and to evaluate its temporal change. For this purpose, we constructed and analysed a co-occurrence network of keywords related to TV commercials by using immense data that include the records of all TV commercials aired in the Kanto area in Japan including Tokyo for a period of 15 years. We found a strong heterogeneity of the co-occurrence relationship, where a few keywords, e.g., 'woman,' 'man','animation', and 'logo', co-occur with a huge number of other keywords every year. A community on a co-occurrence network can be regarded as a set of keywords that are mutually associated with each other through TV commercials. We examined the characteristics of the communities by associating them with categories of advertised products and found a temporal change in which the relationship between the communities possessing the image of entertainment and children and the category of PC and AN gradually increases in strength. However, there was a consistent tendency in the examined period for the product categories related to communities that include 'man' to be less associated with those that include 'woman' and vice versa, which implicates a gender role inequality underlying the various appeals in TV commercials.
\end{abstract}

Keywords: TV commercials, Co-occurrence networks, Community structure, Image structure, Gender role inequality

\section{Introduction}

A network representation is an effective way to determine a hidden characteristic in the objects observed (Newman 2003). For example, we can find several key persons if we construct a network in which each node represents a person and each edge represents the interaction between two individuals, and consider the role of each node (an individual) within the network structure. Various meanings and quantities are assigned to the edges in the network representation, for example, friendship or influence among individuals (Watts and Dodds 2007; Castellano et al. 2009), regulatory relationships between genes or neurons (Gross and Blasius 2008; Socolar and Kauffman 2003), and the correlation between two time series of, for example, stock returns (Mantegna 1999; Mizokami and Ohnishi 2018). The co-occurrence of two items is one of the relationships that can

OThe Author(s), 2021. Open Access This article is licensed under a Creative Commons Attribution 4.0 International License, which permits use, sharing, adaptation, distribution and reproduction in any medium or format, as long as you give appropriate credit to the original author(s) and the source, provide a link to the Creative Commons licence, and indicate if changes were made. The images or other third party material in this article are included in the article's Creative Commons licence, unless indicated otherwise in a credit line to the material. If material is not included in the article's Creative Commons licence and your intended use is not permitted by statutory regulation or exceeds the permitted use, you will need to obtain permission directly from the copyright holder. To view a copy of this licence, visit http:// creativecommons.org/licenses/by/4.0/. 
be represented by an edge (Radhakrishnan et al. 2017; Su and Lee 2010; Zhang et al. 2012; Özgür et al. 2008).

Keyword co-occurrence networks, where nodes denote the keywords in articles, have been constructed and analysed to investigate the knowledge structure in the academic fields (Radhakrishnan et al. 2017; Su and Lee 2010). In these networks, an edge represents the co-occurrence of two keywords in the same article, and an edge occasionally has a weight that represents the frequency of their co-occurrence. A node with a high degree or strength, which is the sum of the weights of edges connected to the focal node, can be regarded as the core of the knowledge structure in the sense that it significantly appears with various topics in the field. Previous studies have investigated such cores in the knowledge structure and their neighbouring nodes to reveal the trends or important topics in the field.

Such a co-occurrence network representation can also be applied to the investigation of the image structure. Ito and Ohnishi (2020) constructed a co-occurrence network of keywords that describe the content of TV commercials and examined the image structure produced by Japanese TV commercials during the last 4 years. Each node in the network is a keyword, and the weight of an edge represents the diversity of the product categories among which two keywords appear in the same commercial. The community structure in the co-occurrence network, where each community can be regarded as a set of images mutually associated through TV commercials, can represent a characteristic of Japanese culture. For example, the keywords 'man' and 'woman', both of which were the cores in the image structure, were assigned to different communities. The community including 'man' was composed of 'laugh', 'talk', and 'office' among other terms, whereas that including 'woman' includes 'look back', 'room', and 'cafe.' Moreover, these communities are related differently to categories of products advertised in commercials.

Indeed, among the extensive studies on the effect of TV commercials (Hekkert et al. 2013; Carreón et al. 2019; Davtyan and Cunningham 2017; Boyland and Halford 2013; Pan 2011), the discussion on the cultural difference of viewer preferences, the appeal of $\mathrm{TV}$ commercials, and the response of viewers to commercials have a long history (Okazaki and Mueller 2007; De Mooij and Hofstede 2011; Moon and Chan 2005; Pham et al. 2013; Liu et al. 2019; Milner and Collins 2000; Bresnahan et al. 2001). Previous studies have classified cultures or countries based on the extent of individualism or collectivism, the strength of uncertainty avoidance or differentiation of male and female roles, and other factors (De Mooij and Hofstede 2011; Okazaki and Mueller 2007). It has been discussed that the feelings of viewers evoked by the items shown in commercials vary significantly according to culture. For example, people in some countries tend to regard direct advertisements as aggressive, whereas people in other countries find them informative (Liu et al. 2019; Okazaki and Mueller 2007; De Mooij and Hofstede 2011). This means that, in turn, the culture of a country can be observed at least partially through TV commercials.

In many investigations conducted on the cultural differences in TV commercials and responses of viewers, there has been an issue of selection bias caused by a limitation or shortage of the collected data (Moon and Chan 2005; Milner and Collins 2000; Bresnahan et al. 2001). The aforementioned study of the image structure of TV commercials (Ito and Ohnishi 2020) addressed this issue by analysing immense amounts of data, 
including all TV commercials aired in the Kanto area of Japan, including Tokyo, from 2017 to 2020, by using the knowledge of complex networks. However, this study was based only on a single network representing keywords co-occurrence in TV commercials from 2017 to 2020 . Here, the question is whether the observed characteristics of the image structure used in the TV commercials in such a study are only attributed to the examined period or are robust for longer periods.

In the present study, we analysed the data of Japanese TV commercials aired over a period 15 years, from 2006 to 2020 . For each year, we constructed a co-occurrence network of keywords describing TV commercials and examined the features of the image structure by analysing the co-occurrence network. We in particular investigated the temporal change of the image structure, i.e., the difference or common features in these co-occurrence networks in the examined years. We conducted a community detection for each year's network and associated communities in networks of consecutive years by evaluating the flow of nodes between them. By the analysis on the temporal change of community structure, we found that the community whose nodes are associated with the keyword 'woman' seems to have a significant relationship to that associated with 'product'. Moreover, the results imply a social issue of gender role inequality: The community whose nodes are associated with 'man' and the community whose nodes are associated with 'woman' exhibited completely different characteristics in the sense of how the nodes in each community are related to the categories of the advertised products during almost all years within the examined period.

\section{Materials and methods}

\section{Data description}

We analysed the data of TV commercials provided by M Data Co., Ltd. (https://mdata. tv/en/). Such data include information on TV commercials aired on five TV stations, Fuji TV, Nippon TV, TBS TV, TV Asahi, and TV Tokyo, in the Kanto area of Japan. The data recorded TV commercials aired from January 1, 2006 to December 31, 2019, and those aired from January 1 to June 30, 2020. The scenario of each commercial is described by keywords such as 'mother and child' and 'nursery'.

Each commercial is classified according to the type of product advertised in the commercial. The classification has three levels, large, middle, and small. We use the large and middle classifications in this study, which are labelled category and subcategory, respectively. Therefore, a product advertised in a commercial is classified into a subcategory, which further belongs to a category. For example, a certain product can be classified into the subcategory of "health drink", which belongs to the category of "drink". Other subcategories belonging to "drink" category are "tea", "fruit juice drink", and so on. Although the number of categories differs in different years according to actually aired commercials, the following categories are used in every year examined: cup noodles, pet food, food, machines, credit cards, finance and insurance, beer, liquor, logistics, communication, car, medicine, snack, oil and tire, infomercial, toy, distribution industry A, distribution industry, detergent, apparel, appliance, household goods, cosmetics, estate, roadshow, AV software, PC and A/V, canned coffee, drink, tobacco, sports, camera and watch, interior, publication, others. Note that though distribution industry and distribution industry A have similar names, they are different categories-the former one 
includes restaurants, retailers, and specialised stores, and the latter one includes convenience stores, department stores, and supermarkets. The number of subcategories also varies in the analysed period. Table 1 shows a summary of the analysed data: the number of commercials, the mean number of co-occurring keywords per commercial, and the numbers of categories and subcategories in each year. Regarding the number of analysed commercials, those that share the same scenario but are aired at different times are counted as different commercials.

\section{Analysis of co-occurrence network of TV commercials and image structure}

We constructed a weighted co-occurrence network $G_{y}$ of the keywords in TV commercials for the year $y \in\{2006,2007, \ldots, 2020\}$. First, for year $y$ and subcategory $\kappa$, we defined an unweighted network $G_{y}^{(\kappa)}$ in which each node represents a keyword, and an edge is drawn between two nodes if they co-occur at least once in the same TV commercial of the product belonging to the subcategory in year $y$ (Fig. 1). Subsequently, we constructed a weighted network $G_{y}$ by merging these unweighted networks $G_{y}^{(\kappa)}$ in year $y$ as follows. The nodes in network $G_{y}$ are all keywords that appear in TV commercials in year $y$, and the weight of an edge between two nodes denotes the proportion of the number of networks for subcategories $G_{y}^{(\kappa)}$ where the edge exists between these two nodes against the total number of subcategories in year $y$. Thus, the weight of an edge between two nodes increases when they co-occur in the same commercials of various types of products.

The resulting network $G_{y}$ indicates that, for example, the nodes of 'mother' and 'child' are connected by an edge with a weight of 0.9 , which shows that these images are frequently used in the same commercial to advertise almost all types of products. Let $A_{i, j}^{y}$ be the weight matrix of $G_{y}$. The strength of node $i, s_{i}=\sum_{j} A_{i, j}^{y}(\in \mathbb{R})$, is the

Table 1 Analysed data

\begin{tabular}{lllll}
\hline Year & \# commercials & $\begin{array}{l}\text { \# co-occurrence } \\
\text { keywords per } \\
\text { commercial }\end{array}$ & \# categories & $\begin{array}{l}\text { \# } \\
\text { subcategories }\end{array}$ \\
\hline 2006 & $1,591,712$ & 3.30 & 39 & 132 \\
2007 & $1,610,762$ & 3.51 & 39 & 129 \\
2008 & $1,590,339$ & 3.64 & 40 & 129 \\
2009 & $1,592,488$ & 3.81 & 40 & 129 \\
2010 & $1,652,888$ & 4.29 & 40 & 130 \\
2011 & $1,657,522$ & 4.42 & 40 & 130 \\
2012 & $1,676,052$ & 4.60 & 40 & 129 \\
2013 & $1,676,414$ & 4.69 & 38 & 128 \\
2014 & $1,672,136$ & 4.71 & 38 & 129 \\
2015 & $1,683,645$ & 5.42 & 40 & 129 \\
2016 & $1,700,535$ & 6.06 & 39 & 130 \\
2017 & $1,682,171$ & 6.57 & 39 & 128 \\
2018 & $1,672,548$ & 7.29 & 38 & 128 \\
2019 & $1,657,577$ & 7.58 & 37 & 37 \\
2020 & 804,827 & 7.37 & 37 & \\
\hline
\end{tabular}

The number of commercials, the mean number of co-occurrence keywords per commercial, and the numbers of categories and subcategories are exhibited for each year 


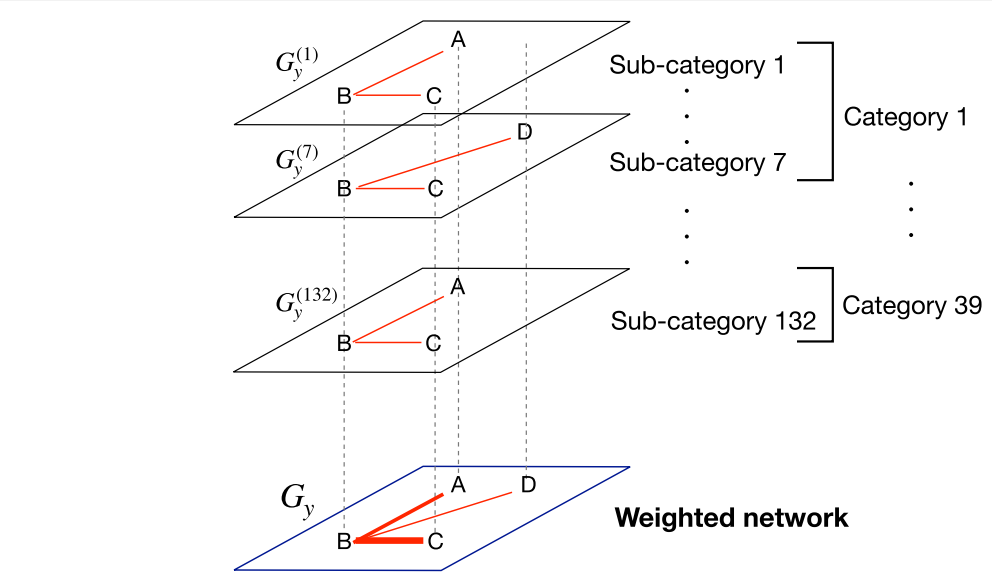

Fig. 1 Schematic image of the construction the co-occurrence network $G_{y}$. The figure was adapted from Ref. (Ito and Ohnishi 2020)

extent to which node $i$ co-occurs with other keywords over various subcategories, whereas the degree $k_{i}$ of node $i$ represents the number of keywords that have cooccurred with node $i$ at least once.

We applied community detection in $G_{y}$ through the modularity maximisation (Fortunato 2010). Modularity $Q$ is defined as follows:

$$
Q=\frac{1}{2 m} \sum_{i, j}\left(A_{i, j}^{y}-\frac{s_{i} s_{j}}{2 m}\right) \delta(c(i), c(j)),
$$

where $2 m=\sum_{i, j} A_{i, j}^{y}, \delta$ denotes Kronecker's delta, and the community to which node $i$ is assigned is denoted as $c(i)$. Because the term $s_{i} s_{j} / 2 m$ in Eq. (1) is the expected weight between nodes $i$ and $j$ in a random network, where the strength distribution is the same as that of $G_{y}$, the modularity $Q$ measures the extent to which nodes within a community are connected tightly compared to the null model for a given graph partition. We obtained a graph partition by (locally) maximising the modularity $Q$ with the Louvain heuristic (Blondel et al. 2008; Aynaud 2020). The Louvain heuristic is a fast algorithm for locally maximising the modularity and is applicable to networks with large sizes, as in our case. We applied the graph partitions 10 times using the Louvain heuristic and adopted the partition that resulted in the highest modularity. In our case, a community consists of keywords that significantly co-occur and are mutually associated over various subcategories.

We also examined the relationship between the communities in $G_{y}$ and the product categories. Note that a node, that is, a keyword, can appear in commercials of multiple (sub)categories, whereas it is assigned to a single community. We first evaluated the extent to which a node is related to each category as follows: Let $N_{i, k}$ be the number of subcategories of category $k$ in which node $i$ appears at least once. For example, the maximum value of $N_{i, k}$ is 7 when there are seven subcategories in category $k$. We normalised $N_{i, k}$ for all nodes appearing in category $k$ as $n_{i, k}:=N_{i, k} / \sum_{j} N_{j, k}$. The value 
of $n_{i, k}$ represents the extent to which node $i$ is related to category $k$. Subsequently, we summed $n_{i, k}$ of nodes belonging to community $l$ as follows:

$$
W_{l, k}=\sum_{\text {node } i \in \text { community } l} n_{i, k}
$$

and normalised it using all nodes belonging to community $l$ and defined as $w_{l, k}$ :

$$
w_{l, k}=\frac{W_{l, k}}{\sum_{j} W_{l, j}}
$$

which represents the extent to which community $l$ is composed of nodes related to category $k$, and thus denotes the strength of the relationship between category $k$ and community $l$.

\section{Results}

\section{Cores of image structure and temporal changes}

In Table 2, we summarise the features of the co-occurrence network $G_{y}$ of the keywords found in TV commercials. The mean degree and the mean strength gradually increase with the year. The reason for this increase may not be attributed to the meaningful change in the characteristics of the TV commercials or of the culture, but presumably to the editorial aspects of the dataset, because the mean number of co-occurrence keywords per commercial continuously increases with the year (Table 1). Rather, it should be noted that we can observe power-law-like distributions in degree and strength in any year, showing a strong heterogeneity (Fig. 2, Additional file 1: Section S.1). Note that the large strength of a node indicates that it has co-occurred with many nodes through TV commercials of various products. By contrast, the degree only represents the number of other nodes that have co-occurred with the node at least once in the same commercial. Therefore, there were a few nodes that co-occurred frequently with other nodes regardless of the variety of advertised products, whereas many nodes co-occurred with a few nodes. This characteristic was robust during the examined period. Figure $2 \mathrm{c}$ shows the relationship between the degree and the strength of each node. We can see a clear tendency in which nodes with a large degree also have a large strength. Hereafter, we regard nodes with a large strength as the cores of the image structure represented by $G_{y}$.

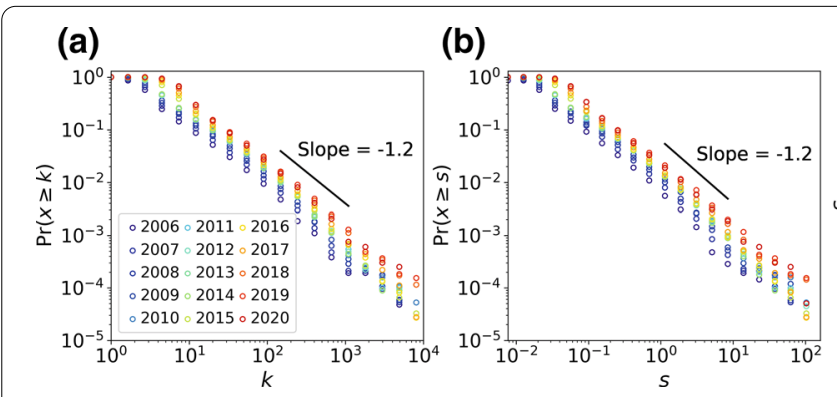

(c)

Fig. 2 Complementary cumulative distribution function (CCDF) of $\mathbf{a}$ the degree $k$ and $\mathbf{b}$ strength $s$ (for the fitting of power law, see Additional file 1: Section S.1). Each colour represents each year. The relationship between the degree and strength of each node is shown in $\mathbf{c}$ 
Table 3 exhibits the nodes having the first to the fifth-largest degree and strength in each year. We can see 'woman' and 'man' in the top-five every year, and thus these nodes can be regarded as robust cores in the image structure of the TV commercials. The keywords 'cinema scope', 'animation', and 'man and woman' were in the higher ranks during the earlier years, but 'product,' 'logo', and 'white back' had higher ranks in the later years.

\section{Temporal change of community structure}

Subsequently, we demonstrate the characteristics of the community structure in the co-occurrence network of TV commercials $G_{y}$ and its temporal changes. The resulting modularity of the graph partition and the number of communities are listed in Table 2.

Table 4 exhibits the node with the largest strength and the size of each community whose size is within the seventh largest during each year. Hereafter, we refer to such a node with the largest strength in each community as a representative node. Core nodes with the largest strength within the whole network, as shown in Table 3, were mostly assigned to different communities, and represented the communities, each of which is a subset of the image structure. Regarding the communities marked with a star in Table 4, seven nodes with the largest strength in each community are shown in Tables 5 and 6 , and the nodes with the largest strength in communities other than those shown in Tables 5 and 6 are shown in Additional file 1: Tables S.2 to S.6.

The diagram in Fig. 3, called the Sankey diagram, visualises the flow of nodes between communities during two consecutive years. Each node represents a community, which is labelled by a representative node, and each flow exhibits the number of nodes that move from the source community to the target community. Herein, we show only communities that have from the first to the seventh-largest size in each year and show only flows between them in the Sankey diagram. In addition, to clarify the mainstream, the flows are removed if their proportion to the total outflow from the source is less than $20 \%$.

Table 2 Feature of the co-occurrence network of each year

\begin{tabular}{llllllll}
\hline Year & \# nodes & \# edges & Density & Mean degree & Mean strength & \# com & $Q$ \\
\hline 2006 & 20,980 & 79,095 & $3.59 \times 10^{-4}$ & 7.54 & 0.06 & 751 & 0.38 \\
2007 & 18,915 & 84,788 & $4.74 \times 10^{-4}$ & 8.97 & 0.08 & 433 & 0.34 \\
2008 & 17,383 & 88,294 & $5.84 \times 10^{-4}$ & 10.16 & 0.09 & 263 & 0.31 \\
2009 & 16,926 & 95,191 & $6.65 \times 10^{-4}$ & 11.25 & 0.11 & 147 & 0.30 \\
2010 & 19,110 & 125,658 & $6.88 \times 10^{-4}$ & 13.15 & 0.12 & 82 & 0.28 \\
2011 & 20,275 & 135,329 & $6.58 \times 10^{-4}$ & 13.35 & 0.12 & 116 & 0.30 \\
2012 & 22,491 & 147,912 & $5.85 \times 10^{-4}$ & 13.15 & 0.12 & 156 & 0.32 \\
2013 & 22,524 & 148,693 & $5.86 \times 10^{-4}$ & 13.20 & 0.12 & 118 & 0.32 \\
2014 & 22,597 & 149,861 & $5.87 \times 10^{-4}$ & 13.26 & 0.12 & 133 & 0.33 \\
2015 & 30,811 & 236,738 & $4.99 \times 10^{-4}$ & 15.37 & 0.14 & 151 & 0.36 \\
2016 & 37,107 & 303,259 & $4.40 \times 10^{-4}$ & 16.35 & 0.14 & 177 & 0.37 \\
2017 & 36,312 & 328,386 & $4.98 \times 10^{-4}$ & 18.09 & 0.16 & 120 & 0.35 \\
2018 & 35,121 & 371,264 & $6.02 \times 10^{-4}$ & 21.14 & 0.19 & 66 & 0.30 \\
2019 & 32,766 & 371,899 & $6.93 \times 10^{-4}$ & 22.70 & 0.21 & 52 & 0.28 \\
2020 & 19,895 & 211,498 & $1.07 \times 10^{-3}$ & 21.26 & 0.20 & 33 & 0.28 \\
\hline
\end{tabular}

The network size, number of edges, edge density, mean degree and strength, number of communities, and modularity of community detection are exhibited 
Table 3 Five nodes with the largest strength during each year

\begin{tabular}{|c|c|c|c|c|c|}
\hline \multirow[t]{2}{*}{2006} & Cinema scope & Woman & Man & Animation & Man and woman \\
\hline & $60.97(5212)$ & 39.34 (3238) & 23.78 (2138) & 21.68 (2153) & 9.14 (930) \\
\hline \multirow[t]{2}{*}{2007} & Cinema scope & Woman & Man & Animation & Man and woman \\
\hline & $68.12(5362)$ & $54.18(3920)$ & $35.74(2813)$ & $24.48(2190)$ & $11.73(1086)$ \\
\hline \multirow[t]{2}{*}{2008} & Cinema scope & Woman & Man & Animation & Man and woman \\
\hline & 74.84 (5571) & 58.29 (4026) & 40.69 (3005) & $25.92(2236)$ & $13.42(1193)$ \\
\hline \multirow[t]{2}{*}{2009} & Cinema scope & Woman & Man & Animation & Man and woman \\
\hline & 99.13 (7045) & $65.67(4385)$ & $47.45(3425)$ & 28.74 (2359) & $16.19(1,385)$ \\
\hline \multirow[t]{2}{*}{2010} & Cinema scope & Woman & Man & Animation & Man and woman \\
\hline & 117.15 (8205) & 87.88 (5809) & $66.08(4752)$ & $29.27(2460)$ & $23.17(1,908)$ \\
\hline \multirow[t]{2}{*}{2011} & Woman & Man & Animation & Cinema scope & Man and woman \\
\hline & $98.23(6441)$ & 70.57 (4964) & 30.46 (2593) & $24.60(2145)$ & $21.87(1,837)$ \\
\hline \multirow[t]{2}{*}{2012} & Woman & Man & Animation & Man and woman & Product \\
\hline & $103.53(7033)$ & 71.06 (5076) & $32.52(2761)$ & $24.54(2057)$ & $22.77(1,938)$ \\
\hline \multirow[t]{2}{*}{2013} & Woman & Man & Animation & Product & Man and woman \\
\hline & 99.20 (6772) & 71.94 (5265) & 33.05 (2787) & 24.95 (2111) & $24.60(2034)$ \\
\hline \multirow[t]{2}{*}{2014} & Woman & Man & Animation & Product & Man and woman \\
\hline & $94.76(6520)$ & 67.91 (5024) & $32.53(271)$ & $24.77(225)$ & $21.20(1832)$ \\
\hline \multirow[t]{2}{*}{2015} & Woman & Man & Product & Animation & Man and woman \\
\hline & 116.56 (8513) & 85.24 (6468) & $52.27(4489)$ & $41.30(3565)$ & $30.27(2612)$ \\
\hline \multirow[t]{2}{*}{2016} & Woman & Man & Product & Animation & Cinema scope \\
\hline & 123.52 (9514) & 86.73 (7096) & $65.33(5656)$ & $46.16(410)$ & $43.98(4100)$ \\
\hline \multirow[t]{2}{*}{2017} & Woman & Product & Man & Cinema scope & Logo \\
\hline & $128.12(9810)$ & 97.50 (8075) & $92.86(7372)$ & $57.67(5086)$ & 53.55 (4768) \\
\hline \multirow[t]{2}{*}{2018} & Woman & Product & Logo & Man & White back \\
\hline & 132.80 (9703) & $132.19(10,539)$ & $123.76(9813)$ & $105.29(8082)$ & $102.80(8133)$ \\
\hline \multirow[t]{2}{*}{2019} & Logo & Product & White back & Woman & Man \\
\hline & $165.55(11,951)$ & $153.51(11,365)$ & $145.06(10,609)$ & 139.83 (9693) & $116.83(8368)$ \\
\hline \multirow[t]{2}{*}{2020} & Logo & Product & White back & Woman & Man \\
\hline & 110.14 (8008) & $100.18(7540)$ & $97.17(7215)$ & 89.66 (6329) & 74.35 (5396) \\
\hline
\end{tabular}

The degree of each node is also shown in parentheses

We found several major streams where many nodes moved together between communities in the examined period using the Sankey diagram. First, we can observe the stream of communities represented by 'woman' during the period from 2006 to 2016 and 'product' from 2017 to 2020, which is called Stream 1. Second, the stream of communities represented by 'animation' for every year except 2019, and by 'black back' in 2019, is also significant, and is called Stream 2. Third, we found a robust stream that consists of communities represented by 'family' and 'eat'. Here, the size of the community represented by 'eat' in 2020 was ranked lower than the seventh largest, and thus it is not shown in the Sankey diagram (the rank was the ninth largest as shown in Additional file 1: Fig. S.5). Considering, however, that the flow from the community represented by 'eat' in 2019 to that represented by 'eat' in 2020 is non-negligible, we set Stream 3 as that configured with the communities represented by 'family', 'family', 'indoor,' 'family', 'eat,' 'eat,' 'family', 'family', 'family', 'eat,' 'eat', 'eat', 'eat', 'eat', and 'eat' in the years from 2006 to 2020, respectively. As mentioned before, the nodes of 'man' and 'woman' were ranked within the top-five highest strength for $G_{y}$ every year. Moreover, we found that these nodes were never assigned to the same community in the analysis period and were the 


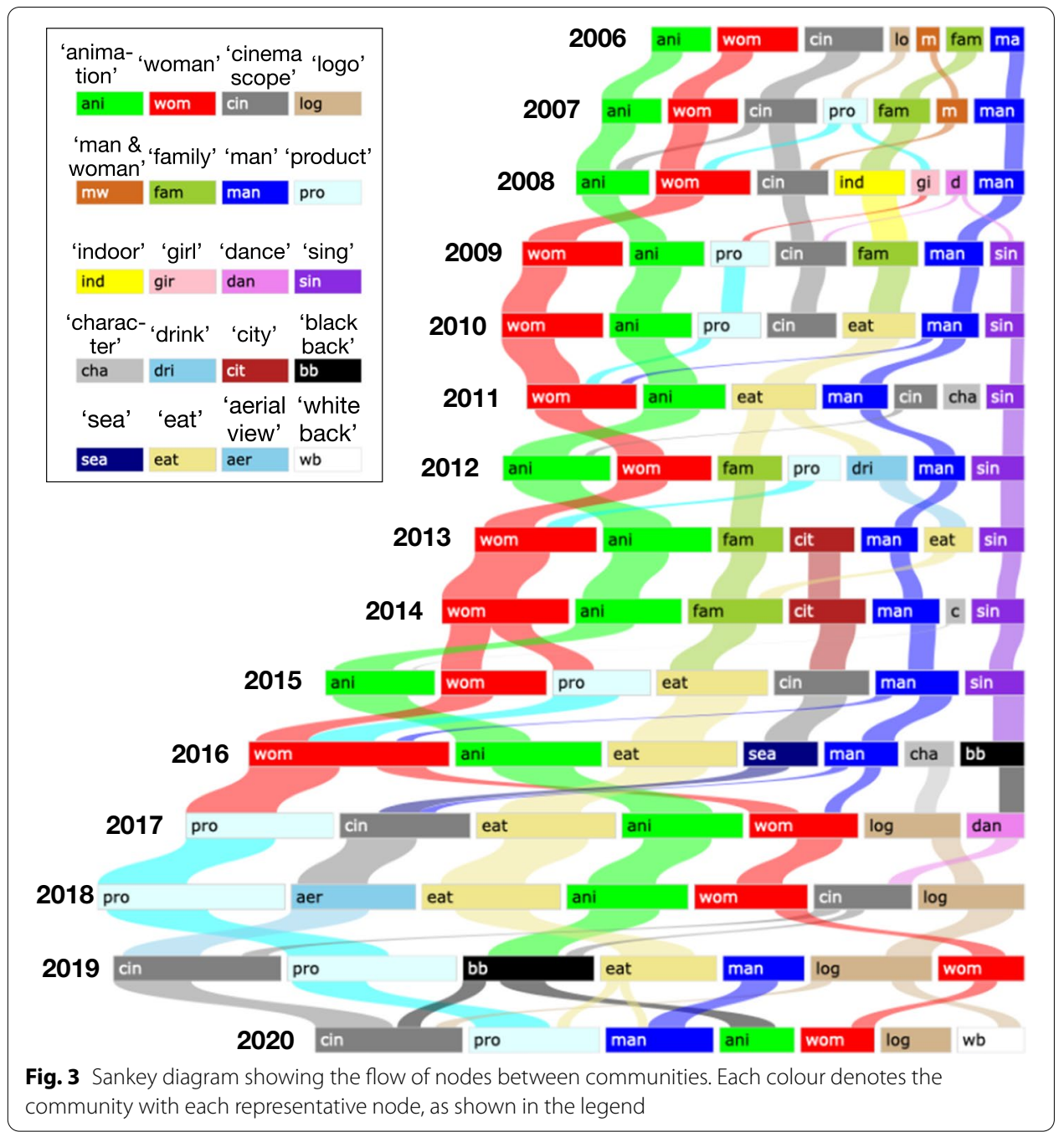

representative nodes in their communities. Therefore, we also observed the following two streams configured by the communities represented by 'man' every year and that represented by 'woman', which are called Streams 4 and 5, respectively. Seven nodes with the largest strength in the communities belonging to Streams 1, 2, and 3 and those belonging to Streams 4 and 5 are shown in Tables 5 and 6 , respectively.

Many nodes in the community represented by 'woman' in 2016 moved to either the community represented by 'product' or the community represented by 'woman' (Fig. 3). Such a split of nodes was presumably caused because 'product' was in the community represented by 'woman' in 2016 (Table 5). The node of 'product' was frequently in the same community as that of 'woman', implicating a significant relationship between the image shared with 'woman' and that with 'product' in TV commercials. In Stream 2, the communities, on the whole, include keywords associated with entertainment, children, and things kids like, e.g. 'animation', 'game screen,' 'boy', and 'girl'. Communities in Stream 3 almost always represented by 'family' and 'eat'. They also include keywords relating to family members and 'kitchen' and 'cooking' and so on. Therefore, it is inferred that the images of family and behaviour related to eating have a strong relationship. Regarding 
Stream 4, the communities represented by 'man' share keywords that evoke a positive expression of feelings or communication such as 'laugh', 'surprise', and 'conversation'. Regarding Stream 5, which is the sequence of communities represented by 'woman', the involved communities were the same as that in Stream 1 until 2016, as mentioned before. It should be noted that the communities in Stream 5 always include 'room' or 'indoor', except in the years 2008, 2019, and 2020.

We further investigated the characteristics of these streams of communities by evaluating the relationship between the communities and the categories, $w_{l, k}$, as described in the Materials and methods section. The heatmaps in Fig. 4 exhibit the value of $w_{l, k}$ in each year for Streams 1, 2, and 3. The horizontal and vertical axes show the category and year, respectively, and we can determine the extent of the relationship $w_{l, k}$ between category $k$ and community $l$ which configures the stream in the year. Figure 5 shows the value of $w_{l, k}$ for Streams 4 and 5 in the same manner as Fig. 4 . The value of $w_{l, k}$ for the other communities with the first to the tenth-largest size in each year are summarised in Additional file 1: Figs S.1 to S.5. In Figs. 4 and 5 , the red (blue) colour indicates that the value of $w_{l, k}$ is higher (lower) than the mean value of $w_{l, k}$ in each stream.

In Streams 1, 2, and 3, we can find unique relationships between the involved communities and categories. Communities in Stream 1 have a strong relationship between categories of medicine, detergent, appliances, household goods, and cosmetics almost every year. By contrast, the values of $w_{l, k}$ in Stream 1 for the categories of infomercial, medicine, detergent, and household goods became more pronounced in the later years, particularly since 2016 or 2017. A reason for this feature in the later years can be inferred as follows. Many nodes that belonged to Stream 1, which originally contains the images of not only 'woman' but also 'product', moved to the community represented by 'product' in 2017 and configured Stream 1 during 2017 to 2020. Images shared by 'product' should be associated with categories of infomercial, medicine, detergent and household goods. The heatmap for Stream 2 exhibits a completely different nature from that of Stream 1. Categories of toy, roadshow, av software, and publication are consistently salient in Stream 2, and PC and A/V gradually increase the extent of the relationship to the stream. These categories related significantly to Stream 2 were less related to Stream 1. Regarding Stream 3, which is associated with the images of 'family' and 'eat', the categories of cup noodle, pet food, food, and appliance are strongly related to the communities in the stream during almost all periods. We can observe that at approximately 2014 and 2015, the relationship of Stream 3 to the categories of beer, liquor, snack, distribution industry $\mathrm{A}$, and distribution industry became strong, whereas those of detergent, household goods, and tobacco became weak. Here, distribution industry (distribution industry A) is a category that includes restaurants, retailers, and specialised stores (convenience stores, department stores, and supermarkets). Therefore, the communities in Stream 3 shifted to a stronger image of foods and drinks in later years.

Figure 5 shows the relationship $w_{l, k}$ between categories $(k)$ and communities $(l)$ represented by 'man' and 'woman', i.e., Streams 4 and 5 , respectively. Interestingly, the value of $w_{l, k}$ tends to be large in stream 4 whereas it is small in stream 5 , and vice versa. For example, although cup noodle and pet food are both food-related, cup noodle is related only to communities represented by 'man' on the whole, and pet food is related only to communities represented by 'woman'. Machine, credit card, finance and insurance, beer, 
(a) Stream 1 (woman, product)

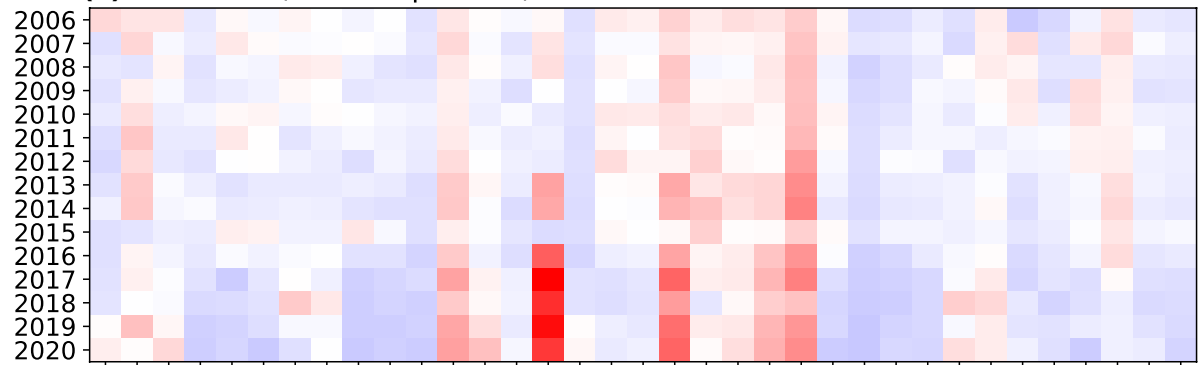

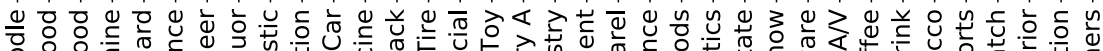

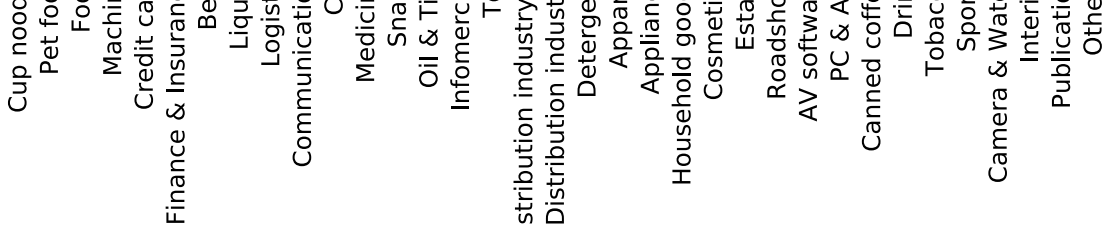

(b) Stream 2 (animation, black back)
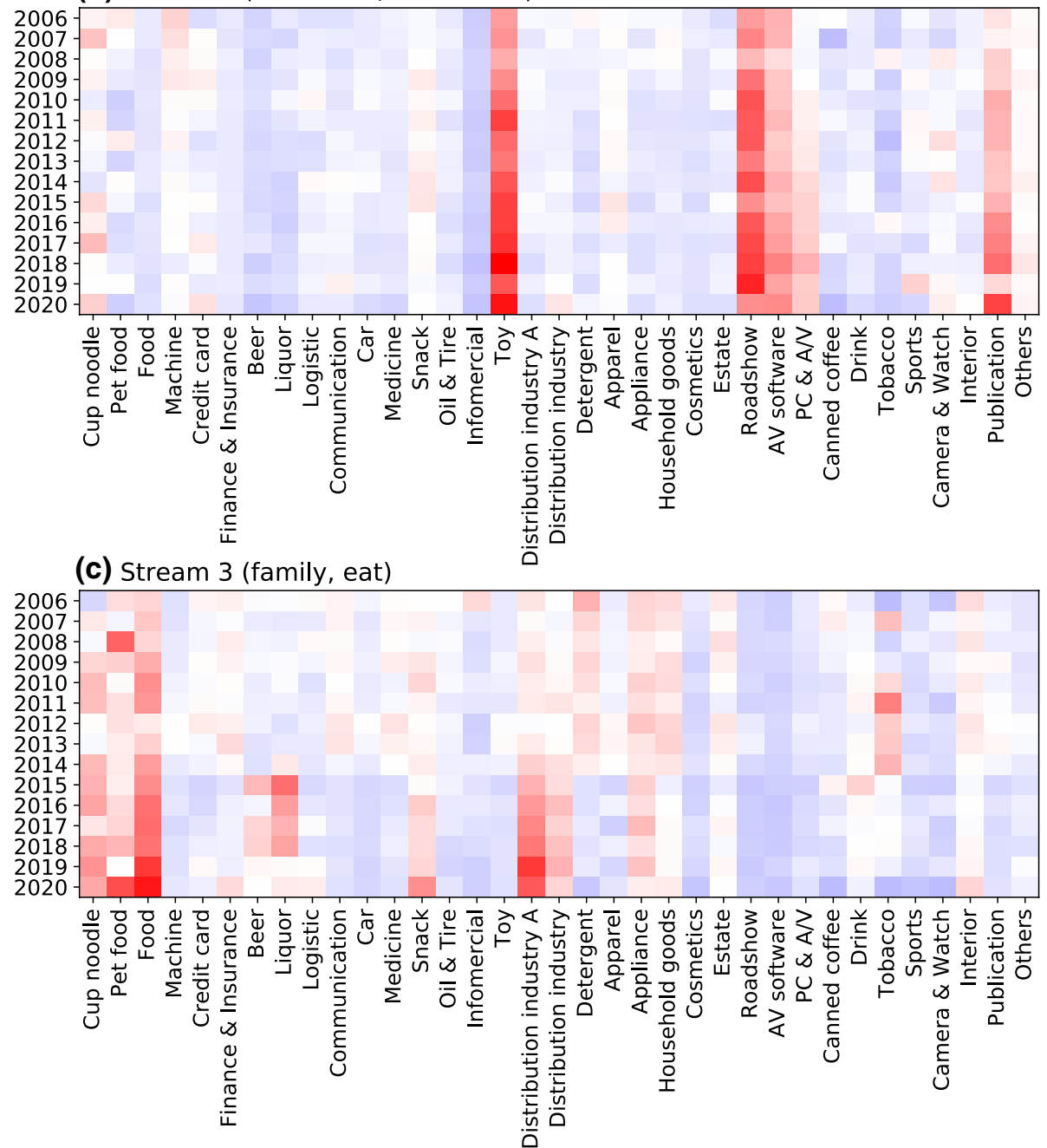

Fig. 4 Relationship $w_{l, k}$ between the categories and the communities included in a Stream 1, b Stream 2, and c Stream 3, for each year 
(a) Stream 4 (man)
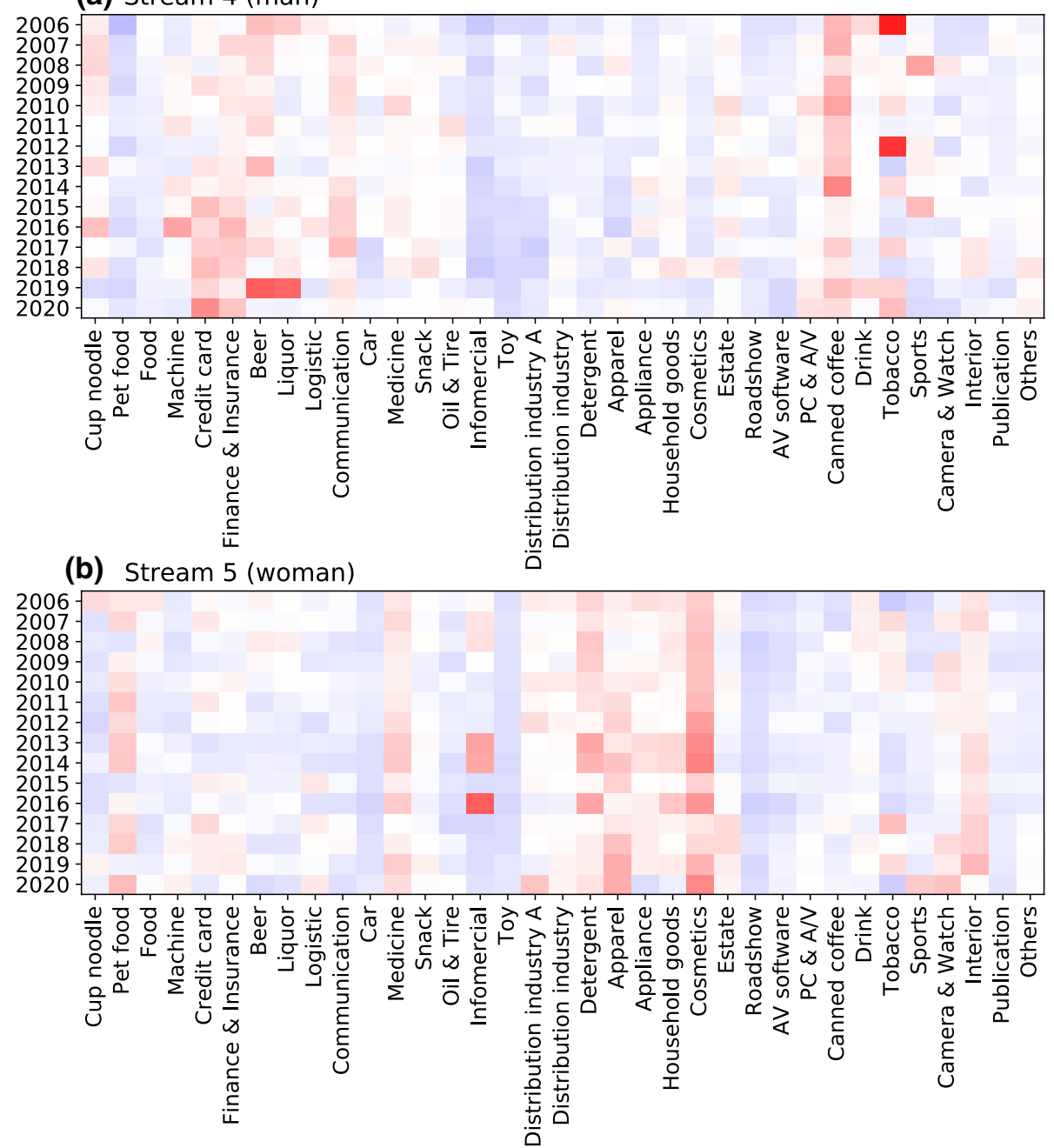

Fig. 5 Relationship $w_{l, k}$ between the categories and the communities included in $\mathbf{a}$ Stream 4 and $\mathbf{b}$ Stream 5 , for each year

communication, canned coffee, and tobacco exhibit strong relationships to Stream 4 of the communities represented by 'man', whereas pet food, medicine, detergent, apparel, appliance, household goods, cosmetics, and interior are strongly related to Stream 5 of the communities represented by 'woman'.

\section{Discussion}

In the literature studying the effects of TV commercials, cultural differences in the appeal of TV commercials have been extensively investigated (Okazaki and Mueller 2007; De Mooij and Hofstede 2011). This suggests, in turn, that we can observe the culture in a country through its TV advertisements. We attempted to reveal the characteristics of the image structure produced based on the appeal in TV advertisements by representing such characteristics through a co-occurrence network of keywords. Here, each node represents a keyword, and the weight of an edge indicates the variety of products of 
Table 4 Community structure

\begin{tabular}{|c|c|c|c|c|c|c|c|}
\hline \multirow[t]{2}{*}{2006} & $\begin{array}{r}\text { Cinema } \\
\text { scope }\end{array}$ & Woman* & Animation* & Man* & Family* & Logo & $\begin{array}{l}\text { Man and } \\
\text { woman }\end{array}$ \\
\hline & 3189 & 2760 & 2219 & 1325 & 1238 & 975 & 911 \\
\hline \multirow[t]{2}{*}{2007} & $\begin{array}{r}\text { Cinema } \\
\text { scope }\end{array}$ & Woman* & Animation* & Man* & Product & Family* & $\begin{array}{l}\text { Man and } \\
\text { woman }\end{array}$ \\
\hline & 2386 & 2114 & 1891 & 1825 & 1762 & 1716 & 1175 \\
\hline \multirow[t]{2}{*}{2008} & Woman* & $\begin{array}{r}\text { Cinema } \\
\text { scope }\end{array}$ & Animation* & Indoor & Man* & Girl & Dance \\
\hline & 2652 & 2566 & 2255 & 2086 & 1607 & 1044 & 737 \\
\hline \multirow[t]{2}{*}{2009} & Woman* & $\begin{array}{r}\text { Cinema } \\
\text { scope }\end{array}$ & Animation* & Product & Man* & Family* & Sing \\
\hline & 2451 & 2382 & 2099 & 1972 & 1775 & 1702 & 1146 \\
\hline \multirow[t]{2}{*}{2010} & Woman* & Product & $\begin{array}{r}\text { Cinema } \\
\text { scope }\end{array}$ & Animation* & Eat* & Man* & Sing \\
\hline & 2966 & 2375 & 2356 & 2335 & 2001 & 1958 & 1606 \\
\hline \multirow[t]{2}{*}{2011} & Woman* & Eat* & Animation* & Man* & Character & $\begin{array}{r}\text { Cinema } \\
\text { scope }\end{array}$ & Sing \\
\hline & 3201 & 2409 & 2390 & 2021 & 1567 & 1528 & 1468 \\
\hline \multirow[t]{2}{*}{2012} & Animation* & Woman* & Sing & Product & Drink & Man* & Family* \\
\hline & 3335 & 2864 & 2112 & 1962 & 1903 & 1837 & 1696 \\
\hline \multirow[t]{2}{*}{2013} & Woman & Animation* & Man* & Family* & City & Sing & Eat \\
\hline & 3702 & 3381 & 2160 & 1984 & 1969 & 1741 & 1563 \\
\hline \multirow[t]{2}{*}{2014} & Woman* & Animation* & Family* & Man* & City & Sing & Character \\
\hline & 3848 & 3317 & 2700 & 2196 & 1955 & 1787 & 1214 \\
\hline \multirow[t]{2}{*}{2015} & Animation* & Woman* & $\begin{array}{r}\text { Cinema } \\
\text { scope }\end{array}$ & Eat* & Product & Man* & Sing \\
\hline & 4144 & 3394 & 3364 & 3226 & 3139 & 2940 & 2363 \\
\hline \multirow[t]{2}{*}{2016} & Woman* & Animation* & Eat* & Man* & Black back & Sea & Character \\
\hline & 6320 & 5598 & 3975 & 2795 & 2768 & 2458 & 2152 \\
\hline \multirow[t]{2}{*}{2017} & Animation* & Product* & Eat* & $\begin{array}{r}\text { Cinema } \\
\text { scope }\end{array}$ & Logo & Woman* & Dance \\
\hline & 4612 & 4569 & 3934 & 3824 & 3776 & 3573 & 2428 \\
\hline \multirow[t]{2}{*}{2018} & Product* & Animation* & Eat* & Logo & $\begin{array}{r}\text { Cinema } \\
\text { scope }\end{array}$ & Woman* & Aerial view \\
\hline & 5369 & 4091 & 3828 & 3608 & 3546 & 3450 & 3286 \\
\hline \multirow[t]{2}{*}{2019} & product* & $\begin{array}{r}\text { Cinema } \\
\text { scope }\end{array}$ & BlackBack* & Logo & Man* & Eat* & Woman* \\
\hline & 4590 & 4471 & 4265 & 4143 & 3414 & 3087 & 2466 \\
\hline \multirow[t]{2}{*}{2020} & $\begin{array}{r}\text { Cinema } \\
\text { scope }\end{array}$ & Product* & Man* & Logo & Animation* & White back & Woman* \\
\hline & 3147 & 2627 & 2576 & 2035 & 1832 & 1529 & 1418 \\
\hline
\end{tabular}

Node with the highest strength in the community for each year and each community that has the first to the seventh largest size during the year. For the communities marked with a star, the other six nodes with large strengths are also shown in Tables 5 and 6

which two keywords co-occur in the same commercial. Therefore, a community can be regarded as a set of keywords that frequently co-occur in various commercials. In particular, the present study investigated how the features of such a co-occurrence network have temporally changed. Our analysis captured a temporal change of the image structure, in which the relationship between communities associated with entertainment and children and the category of PC and $\mathrm{A} / \mathrm{V}$ gradually increases. By contrast, the 
Table 5 Seven nodes with the largest strength in each community involved in Streams 1, 2 and 3

\begin{tabular}{|c|c|c|}
\hline Stream & Year & Nodes \\
\hline & 2006 & Woman, walk, eat, inside a store, sofa, stairs, office \\
\hline & 2007 & Woman, indoor, photo, PC, sofa, bicycle, sit \\
\hline & 2008 & Woman, product, drink, tell, inside a store, small screen, stairs \\
\hline & 2009 & Woman, indoor, drink, tell, dog, mobile phone, PC \\
\hline & 2010 & Woman, tell, indoor, walk, room, mobile phone, city \\
\hline & 2011 & Woman, indoor, walk, room, city, PC, dog \\
\hline Stream 1 & 2012 & Woman, indoor, walk, room, inside a store, pose, stairs \\
\hline 'Woman' & 2013 & Woman, product, indoor, room, paint, cafe, stairs \\
\hline \multirow[t]{13}{*}{ 'Product' } & 2014 & Woman, product, indoor, walk, impression, stairs, paint \\
\hline & 2015 & Woman, indoor, walk, smile, room, flower, fireworks \\
\hline & 2016 & Woman, product, tell, indoor, walk, photo, smile \\
\hline & 2017 & Product, tell, smile, studio, close-up a face, round frame, a word \\
\hline & 2018 & Product, man and woman, drink, pose, surprise, tell, illustration \\
\hline & 2019 & Product, indoor, a word, round frame, product in hand, description, studio \\
\hline & 2020 & $\begin{array}{l}\text { Product, product in hand, round frame, blue back, another product, } \\
\text { impression, image }\end{array}$ \\
\hline & 2006 & Animation, run, boy, C.G., fly, flame, fight \\
\hline & 2007 & Animation, boy, run, C.G., fight, cry, flame \\
\hline & 2008 & Animation, run, boy, illustration, fight, explosion, forest \\
\hline & 2009 & Animation, girl, run, boy, flame, explosion, C.G. \\
\hline & 2010 & Animation, girl, run, boy, character, card, flame \\
\hline & 2011 & Animation, girl, boy, run, character, game screen, C.G. \\
\hline Stream 2 & 2012 & Animation, cinema scope, girl, run, boy, character, card \\
\hline 'Animation' & 2013 & Animation, cinema scope, girl, character, logo, boy, run \\
\hline \multirow[t]{13}{*}{ 'Black back' } & 2014 & Animation, cinema scope, character, logo, boy, run, card \\
\hline & 2015 & Animation, logo, girl, boy, character, CG, game screen \\
\hline & 2016 & Animation, run, girl, boy, game screen, character, illustration \\
\hline & 2017 & Animation, girl, character, game screen, boy, illustration, pose \\
\hline & 2018 & Animation, white back logo, girl, character, boy, shout, game screen \\
\hline & 2019 & Black back, animation, white back logo, run, girl, character, boy \\
\hline & 2020 & Animation, girl, character, boy, card, game screen, flame \\
\hline & 2006 & Family, married couple, mother, child, daughter, father, wife \\
\hline & 2007 & Family, eat, child, mother, daughter, father, mother and a child \\
\hline & 2008 & Indoor, family, child, eat, photo, mother, married couple \\
\hline & 2009 & Family, eat, child, married couple, mother, boy, girl \\
\hline & 2010 & Eat, family, child, mother, married couple, photo, boy \\
\hline & 2011 & Eat, family, child, married couple, mother, inside a store, girl \\
\hline Stream 3 & 2012 & Family, child, mother, girl, boy, dog, father \\
\hline 'Family' & 2013 & Family, married couple, child, mother, boy, conversation, photo \\
\hline \multirow[t]{7}{*}{ 'Eat' } & 2014 & Family, eat, married couple, mother, child, inside a store, taste \\
\hline & 2015 & Eat, drink, family, surprise, married couple, inside a store, kitchen \\
\hline & 2016 & Eat, family, married couple, red back, girl, child, kitchen \\
\hline & 2017 & Eat, family, laugh, married couple, cooking, parent and child, inside a store \\
\hline & 2018 & Eat, family, laugh, parent and child, cooking, child, kitchen \\
\hline & 2019 & Eat, family, photo, parent and child, cooking, kitchen, inside a store \\
\hline & 2020 & Eat, baby, field, white back,logo, cut, sleep, curry \\
\hline
\end{tabular}


Table 6 Seven nodes with the largest strength in each community involved in Streams 4 and 5

\begin{tabular}{|c|c|c|}
\hline Stream & Year & Nodes \\
\hline & 2006 & Man, drink, tell, train, rooftop, surprise, building \\
\hline & 2007 & Man, tell, office, TV, look, bench, still image \\
\hline & 2008 & Man, sea, bicycle, laugh, store, rooftop, desert \\
\hline & 2009 & Man, TV, office, phone, class room, meeting, speak \\
\hline & 2010 & Man, office, suited man, stairs, speak, window, question \\
\hline & 2011 & Man, black and white, office, kimono woman, laugh, surprise, greeting \\
\hline Stream 4 & 2012 & Man, office, surprise, phone, greeting, another man, studio \\
\hline \multirow[t]{14}{*}{ 'Man' } & 2013 & Man, black and white, surprise, phone, laugh, another man, question \\
\hline & 2014 & Man, office, conversation, phone, park, question, meeting \\
\hline & 2015 & Man, office, conversation, laugh, phone, cafe, cry \\
\hline & 2016 & Man, office, laugh, bicycle, greeting, phone, park \\
\hline & 2017 & Man, another man, cat, izakaya, in a bus, Japanese room, call \\
\hline & 2018 & Man, speak, another man, cafe, pointing, man, close-up, worried \\
\hline & 2019 & Man, drink, laugh, surprise, speak, conversation, tell \\
\hline & 2020 & Man, man and woman, a word, surprise, pose, speak, laugh \\
\hline & 2006 & Woman, walk, eat, inside a store, sofa, stairs, office \\
\hline & 2007 & Woman, indoor, photo, PC, sofa, bicycle, sit \\
\hline & 2008 & Woman, product, drink, tell, inside a store, small screen, stairs \\
\hline & 2009 & Woman, indoor, drink, tell, dog, mobile phone, PC \\
\hline & 2010 & Woman, tell, indoor, walk, room, mobile phone, city \\
\hline & 2011 & Woman, indoor, walk, room, city, PC, dog \\
\hline Stream 5 & 2012 & Woman, indoor, walk, room, inside a store, pose, stairs \\
\hline \multirow[t]{8}{*}{ 'Woman' } & 2013 & Woman, product, indoor, room, paint, cafe, stairs \\
\hline & 2014 & Woman, product, indoor, walk, impression, stairs, paint \\
\hline & 2015 & Woman, indoor, walk, smile, room, flower, fireworks \\
\hline & 2016 & Woman, product, tell, indoor, walk, photo, smile \\
\hline & 2017 & Woman, indoor, walk, office, dog, wave a hand, stairs \\
\hline & 2018 & Woman, smile, walk, indoor, sky, dog, photograph \\
\hline & 2019 & Woman, smile, pose, office, turn around, sit, cafe \\
\hline & 2020 & Woman, walk, another woman, step, jump, turn around, sit \\
\hline
\end{tabular}

relationship between the categories and Stream 4 ('man') exhibited a different nature from that of Stream 5 ('woman'), and this feature was consistent during the period examined.

The power law in terms of degree or strength distribution has been found in networks representing various phenomena, such as social interaction and citation relationships, collaboration relationships or the co-occurrence of keywords in the academic literature (Newman 2003; Castellano et al. 2009; Karimi et al. 2019; Zhang et al. 2012). We also observed a power law or a strong heterogeneity in the co-occurrence relationship in the keywords from TV commercials. The strength of the keywords shown in Table 3, e.g. 'woman' and 'man', are large. These nodes are tightly connected to, that is, strongly associated with, other nodes through TV commercials. These nodes with high strength can be regarded as cores in the image structure produced by the TV commercials. In the later years of the examined period, the degree and strength of the keyword 'logo' became the largest, whereas they were not within the top five largest in earlier years. Studies investigating culture and TV commercials have pointed out that displaying the 
corporate identity logo is one of the features of Japanese commercials, where the establishment of trust between the company and consumers has a significant effect on consumer attitudes toward the products (De Mooij and Hofstede 2011). According to the data provider, the increase of the strength of 'logo' seems to be attributed to the change in their editorial policy needed considering the importance of this keyword. Temporal changes in the community structure in the co-occurrence network seem to capture the evolution of technology during the analysed period as well. Stream 2 is configured by communities represented by 'animation' (or by 'black back' in 2019) and is associated with keywords that evoke entertainment and aspects enjoyed by children. The relationship between this stream and the category of $\mathrm{PC}$ and $\mathrm{A} / \mathrm{V}$ strengthens with each year. This presumably indicates the situation in which PC becomes a more common tool than ever before when enjoying hobbies or when children play.

Our analysis reveals not only such temporal changes, but also robust characteristics in Japanese culture. We found a significant inequality in Streams 4 and 5, configured with the communities represented by 'man' and 'woman', respectively, regarding their relationship to the various categories. Categories that had a strong relationship with the communities represented by 'man' had mostly a weak relationship to that represented by 'woman' and vice versa. The extent to which male and female roles are differentiated is an indicator characterising a culture in studies conducted on cultural differences in commercials. Our result is consistent with previous studies that showed the segregation of male and female roles in Japanese commercials, which are conducted around the year 2000 (Bresnahan et al. 2001; Milner and Collins 2000). Moreover, we found that the communities represented by 'woman' have a strong relationship to the image of 'product.' Considering the keywords included in Stream 1, e.g. 'woman', 'product', 'indoor', 'room, and 'stairs', and considering the strong relationship of the communities in this stream to the categories of medicine, detergent, household goods, cosmetics and so on, we can infer that a situation in which women actually use a product indoors is one of the significant images in TV commercials.

A strength of our study is the use of large data which records not only advertised products or aired time but also keywords representing the content in each TV commercial. The data covers all commercials aired in the Kanto area, including Tokyo, during the last 15 years. Many previous studies on cultural differences in TV commercials performed content analysis, which takes significant effort, e.g. coders rated the content of commercials according to various scales or checked the presence of items relating to the studies (Okazaki and Mueller 2007). Presumably because of this large effort, analysed commercials in the previous studies were limited to those aired in a single day or only during primetime for a week (Milner and Collins 2000; Bresnahan et al. 2001). Therefore, the findings in their analyses could be limited to the analysed seasons or period. In this study, we did not have to consider the effect of such selection bias on the results of the image structure produced by TV commercials and were able to observe an entire image underlying Japanese commercials. Although some of our findings on the characteristics of Japanese commercials are consistent with those in previous reports, the results of our study supported by such a large coverage of data should be more persuasive.

In that sense, gender role inequality, which is one of the implications of our analysis on TV commercials, should be a significant social issue. Analyses of large data have found 
gender inequality in various platforms and systems, such as in Wikipedia and crowdfunding, or in academic collaborations (Wagner et al. 2016; Horvát and Papamarkou 2017; Jadidi et al. 2018; Karimi et al. 2019). TV commercials are created to generate purchase intention for products or a positive attitude toward a brand; these motivations are unique to commercials and differ from those of other platforms, such as Wikipedia. That data with different reasons can reveal the same issue should be of interest to researchers. Our research should contribute to showing the diversity of large data uncovering social issues, as well as previous studies.

Here, we will discuss the technical aspects of our analysis. The resolution limit is an issue in community detection, which means that we cannot obtain communities whose size is relatively small when we conduct a graph partition through a modularity maximisation (Fortunato 2010). Therefore, the resolution limit can make us fail to find a group of nodes where the size is small but the nodes are mutually and tightly connected, which can indeed be called a 'community' in a social network. In our case, the resolution limit may not be a significant issue because what we attempted to observe is a rough image or an overview underlying the image structure produced by TV commercials, and even though we found a group consisting of a small number of nodes that are tightly connected, this might represent a trivial combination of keywords. However, as a future perspective, it would be interesting to compare the community structures obtained at various resolutions. By doing so, we may be able to observe in detail the streams of nodes among communities in years and help us better understand the detailed transition of our culture.

In addition, we may obtain further insight about the culture by analysing TV commercials in each season or area. Japanese seasons are distinct from each other, and there are many traditional events throughout the year. Moreover, the culture and value vary in different areas, and aired TV commercials are different in Japan. We may be able to find the effect of traditional or modern cultures on TV commercials by comparing commercials in various areas and in different seasons.

\section{Supplementary Information}

The online version contains supplementary material available at https://doi.org/10.1007/s41109-021-00393-4.

Additional file 1. Supplementary information

Acknowledgements

Not applicable.

Authors' contributions

MII and TO contributed conception and design of the study; MII and TO performed the statistical analysis; MII wrote the first draft of the manuscript; MII and TO wrote sections of the manuscript. Both authors contributed to manuscript revision, read and approved the submitted version.

Funding

This work was supported by JSPS KAKENHI Grant Number JP20K19929 and JP19H01114.

\section{Availability of data and materials}

The data that support the findings of this study are available from M Data Co., Ltd. (https://mdata.tv/en/) but restrictions apply to the availability of these data, which were used under license for the current study, and so are not publicly available. Data are however available from the authors upon reasonable request and with permission of M Data Co., Ltd.

Declaration

Competing interests

The authors declare that they have no competing interests. 
Received: 8 March 2021 Accepted: 29 June 2021

Published online: 15 July 2021

\section{References}

Aynaud T (2020) python-louvain 0.14: Louvain algorithm for community detection. https://github.com/taynaud/pythonlouvain

Blondel VD, Guillaume J-L, Lambiotte R, Lefebvre E (2008) Fast unfolding of communities in large networks. J Stat Mech 10:10008. https://doi.org/10.1088/1742-5468/2008/10/P10008

Boyland EJ, Halford JCG (2013) Television advertising and branding. Effects on eating behaviour and food preferences in children. Appetite 62:236-241. https://doi.org/10.1016/j.appet.2012.01.032

Bresnahan MJ, Inoue Y, Liu WY, Nishida T (2001) Changing gender roles in prime-time commercials in Malaysia, Japan, Taiwan, and the United States. Sex Roles 45(1):117-131. https://doi.org/10.1023/A:1013068519583

Carreón ECA, Nonaka H, Hentona A, Yamashiro H (2019) Measuring the influence of mere exposure effect of TV commercial adverts on purchase behavior based on machine learning prediction models. Inf Process Manag 56(4):1339-1355. https://doi.org/10.1016/j.ipm.2019.03.007

Castellano C, Fortunato S, Loreto V (2009) Statistical physics of social dynamics. Rev Mod Phys 81(2):591. https://doi.org/10. 1103/RevModPhys.81.591

Davtyan D, Cunningham I (2017) An investigation of brand placement effects on brand attitudes and purchase intentions: brand placements versus TV commercials. J Bus Res 70:160-167. https://doi.org/10.1016/j.jbusres.2016.08.023

De Mooij M, Hofstede G (2011) Cross-cultural consumer behavior: a review of research findings. J Int Consum Mark 23(3-4):181-192. https://doi.org/10.1080/08961530.2011.578057

Fortunato S (2010) Community detection in graphs. Phys Rep 486(3):75-174. https://doi.org/10.1016/.jphysrep.2009.11.002

Gross T, Blasius B (2008) Adaptive coevolutionary networks: a review. J R Soc Interface 5(20):259-271. https://doi.org/10.1098/ rsif.2007.1229

Hekkert P, Thurgood C, Whitfield TA (2013) The mere exposure effect for consumer products as a consequence of existing familiarity and controlled exposure. Acta Psychol 144(2):411-417. https:/doi.org/10.1016/j.actpsy.2013.07.015

Horvát E-A, Papamarkou, T (2017) Gender differences in equity crowdfunding. In: Proceedings of the AAAl conference on human computation and crowdsourcing, vol 5. https://ojs.aaai.org/index.php/HCOMP/article/view/13319

Ito MI, Ohnishi T (2020) Uncovering the image structure of Japanese TV commercials through a co-occurrence network representation. In: Benito RM, Cherifi C, Cherifi H, Moro E, Rocha LM, Sales-Pardo M (eds) Complex networks and their applications IX. Ccomplex networks 2020. Studies in computational intelligence, vol 943. Springer, Berlin, pp 273-283. https://doi.org/10.1007/978-3-030-65347-7_23

Jadidi M, Karimi F, Lietz H, Wagner C (2018) Gender disparities in science? Dropout, productivity, collaborations and success of male and female computer scientists. Adv Complex Syst 21 (03n04):1750011. https://doi.org/10.1142/S02195259175001 14

Karimi F, Mayr P, Momeni F (2019) Analyzing the network structure and gender differences among the members of the networked knowledge organization systems (NKOS) community. Int J Digit Libr 20(3):231-239. https://doi.org/10.1007/ s00799-018-0243-0

Liu F, Kanso A, Zhang Y, Olaru D (2019) Culture, perceived value, and advertising acceptance: a cross-cultural study on mobile advertising. J Promot Manag 25(7):1028-1058. https://doi.org/10.1080/10496491.2019.1612495

Mantegna RN (1999) Hierarchical structure in financial markets. Eur Phys J B 11(1):193-197. https://doi.org/10.1007/s 1005 10050929

Milner LM, Collins JM (2000) Sex-role portrayals and the gender of nations. J Advert 29(1):67-79. https://doi.org/10.1080/ 00913367.2000 .10673604

Mizokami C, Ohnishi T (2018) Revealing persistent structure of international trade by nonnegative matrix factorization. In: Cherifi C, Cherifi H, Karsai M, Musolesi M (eds) Complex networks and their applications VI. Complex networks 2017. Studies in computational intelligence, vol 689. Springer, Berlin, pp 1088-1099. https://doi.org/10.1007/978-3-319-721507_88

Moon YS, Chan K (2005) Advertising appeals and cultural values in television commercials A comparison of Hong Kong and Korea. Int Mark Rev 22(1):48-66. https://doi.org/10.1108/02651330510581172

Newman MEJ (2003) The structure and function of complex networks. SIAM Rev 45(2):167-256

Okazaki S, Mueller B (2007) Cross-cultural advertising research: where we have been and where we need to go. Int Mark Rev 24(5):499-518. https://doi.org/10.1108/02651330710827960

Özgür A, Cetin B, Bingol H (2008) Co-occurrence network of Reuters news. Int J Mod Phys C 19(05):689-702. https://doi.org/ $10.1142 / 50129183108012431$

Pan S (2011) The role of TV commercial visuals in forming memorable and impressive destination images. JTravel Res 50(2):171-185. https://doi.org/10.1177/0047287509355325

Pham MT, Geuens M, De Pelsmacker P (2013) The influence of ad-evoked feelings on brand evaluations: empirical generalizations from consumer responses to more than 1000 TV commercials. Int J Res Mark 30(4):383-394. https://doi.org/10. 1016/j.jijesmar.2013.04.004

Radhakrishnan S, Erbis S, Isaacs JA, Kamarthi S (2017) Novel keyword co-occurrence network-based methods to foster systematic reviews of scientific literature. PLoS ONE 12(3):0172778. https://doi.org/10.1371/journal.pone.0172778

Socolar JES, Kauffman SA (2003) Scaling in ordered and critical random Boolean networks. Phys Rev Lett 90(6):068702. https:// doi.org/10.1103/PhysRevLett.90.068702

Su H-N, Lee P-C (2010) Mapping knowledge structure by keyword co-occurrence: a first look at journal papers in technology foresight. Scientometrics 85(1):65-79. https://doi.org/10.1007/s11192-010-0259-8

Wagner C, Graells-Garrido E, Garcia D, Menczer F (2016) Women through the glass ceiling: gender asymmetries in Wikipedia. EPJ Data Sci 5:1-24. https://doi.org/10.1140/epjds/s13688-016-0066-4 
Watts DJ, Dodds PS (2007) Influentials, networks, and public opinion formation. J Consum Res 34(4):441-458. https://doi.org/ $10.1086 / 518527$

Zhang J, Xie J, Hou W, Tu X, Xu J, Song F, Wang Z, Lu Z (2012) Mapping the knowledge structure of research on patient adherence: knowledge domain visualization based co-word analysis and social network analysis. PLOS ONE 7(4):34497. https://doi.org/10.1371/journal.pone.0034497

\section{Publisher's Note}

Springer Nature remains neutral with regard to jurisdictional claims in published maps and institutional affiliations.

Submit your manuscript to a SpringerOpen ${ }^{\circ}$ journal and benefit from:

- Convenient online submission

- Rigorous peer review

- Open access: articles freely available online

- High visibility within the field

- Retaining the copyright to your article

Submit your next manuscript at $>$ springeropen.com 